\title{
Die Anregungsenergie der isomeren Zustände des Rhodiums 103 und 104
}

\author{
Von Arnold Flammersfeld und Otto Bruna \\ Aus dem Kaiser-Wilhelm-Institut für Chemie, Tailfingen \\ (Z. Naturforschg. 2 a, 241-244 [1947]; eingegangen am 26. Januar 1947)
}

\begin{abstract}
Die Anregungsenergien der isomeren Zustände des Rhodiums wurden durch Messung der Reichweite der Umwandlungselektronen bestimmt. Die vom ${ }^{104} \mathrm{Rh}^{*}(T=4,3 \mathrm{~min})$ ausgesandten Sekundärelektronen haben eine Reichweite von $8,9 \mathrm{mg} / \mathrm{cm}^{2} \mathrm{Al}$ entsprechend einer Energie von $87 \mathrm{KeV}$. Der Drehimpulsunterschied wird auf $\Delta i=4$ geschätzt, und da deshalb der Hauptteil der Elektronen in der $L$-Schale und noch merkbare Anteile in der $(M+N)$-Schale ausgelöst werden dürften, beträgt die Anregungsenergie des angeregten Zustandes $87 \mathrm{KeV}$. Aus der Intensität der Elektronen wird geschlossen, daß der Isomerenübergang zu 100\% durch Aussendung dieser Sekundärelektronen vor sich geht und daß kein direkter Übergang des ${ }^{104} \mathrm{Rh}^{*}$ in ${ }^{104} \mathrm{Pd}$ stattfindet.

${ }_{103} \mathrm{Rh}^{*}$, das aus dem stabilen 103Rh durch Anregung mit schnellen Neutronen zu erhalten ist, hat die Halbwertszeit $T=52 \pm 2$ min und sendet Umwandlungselektronen der Reichweite $2,1 \mathrm{mg} / \mathrm{cm}^{2}$ aus. Da die Drehimpulsdifferenz auf $\Delta i=4$ geschätzt wird, ist die Energie der Elektronen gleich der Anregungsenergie; diese beträgt $42 \mathrm{KeV}$. Die bereits früher aufgefundene Quantenstrahlung ist Röntgenstrahlung.
\end{abstract}

$\mathrm{B}_{\mathrm{s}}^{\mathrm{e}}$ ei Rhodium sind zwei Fälle von Isomerie sichergestellt, ${ }^{104} \mathrm{Rh}^{*}(T=4,3 \mathrm{~min})$ und ${ }^{103} \mathrm{Rh}^{*}$ $(T=52 \mathrm{~min})$. Beim ersteren liegen über die Anregungsenergie nur ungenaue und beim letzteren gar keine Angaben vor; es sollte daher im folgenden die Energie beider Isomere bestimmt werden.

\section{1. ${ }^{104} \mathrm{Rh} *$}

Rhodium zeigt bei Bestrahlung mit langsamen Neutronen zwei Aktivitäten von 41,8 sec und 4,34 min Halbwertszeit ${ }^{1}$, die nach $\mathrm{P}$ on tec or vo ${ }^{2}$ isomeren Zuständen des Isotops ${ }^{104} \mathrm{Rh}$ zugeordnet werden. Pontecorvo hat gefunden, daß das scheinbar von der 4,3 min-Aktivität herrührende $\beta$ Kontinuum, das mit dem von der 42 -sec-Aktivität übereinstimmt, von einer Gruppe von Elektronen überlagert ist, deren Energie etwa 35 bis $60 \mathrm{KeV}$ beträgt und deren Intensität etwa $30 \%$ zu sein schien, während die 42-sec-Aktivität keine derartige zusätzliche Gruppe zeigt. Mit P o n te c or vo ist daraus zu schließen, daß die 42-sec-Aktivität dem Grundzustand ${ }^{104} \mathrm{Rh}$ und die 4,3-min-Aktivität dem angeregten Zustand ${ }^{104} \mathrm{Rh}^{*}$ zugehört, welcher mindestens zum Teil $(30 \%)$ in den Grund-

1 E. Amaldi, O. D'Agostino, E. Fermi, B. Pontecorvo, F. Rasetti u. E. Segré, Proc. Roy. Soc. [London] Ser. A 149, 522 [1935]. zustand übergeht, dabei durch innere Umwandlung an Stelle von $\gamma$-Strahlung die genannten Elektronen aussendet und dann erst durch $\beta$-Zerfall mit 42 sec Halbwertszeit in ${ }^{104} \mathrm{Pd}$ übergeht. Diese Deutung wurde gestützt durch Untersuchung der zugehörigen $\beta$-Spektren durch E. C. Critten d en jr. ${ }^{3}$. Würde der angeregte Zustand ${ }^{104} \mathrm{Rh}^{*}$ direkt durch $\beta$-Zerfall in $\mathrm{Pd}$ übergehen, so müßte wegen des zu erwartenden größeren Spinunterschiedes dieser $\beta$ Zerfall einem verbotenen Übergang entsprechen und das $\beta$-Kontinuum voraussichtlich eine andere Form zeigen als das des 42-sec-Körpers, der nach Ausweis des Sargent-Diagramms ein erlaubter Übergang ist. Innerhalb der Versuchsfehler (W il son-Kammer) fand Crittenden die beiden $\beta$ Spektren gleich.

Das Ziel der vorliegenden Untersuchung war einmal, die Energiedifferenz des isomeren Zustandes zum Grundzustand durch Messung der Energie der Umwandlungselektronen genauer zu bestimmen, und ferner aus der Intensität dieser Elektronen Aufschluß zu gewinnen, ob der angeregte Zustand ${ }^{104} \mathrm{Rh}^{*}$ vor dem $\beta$-Zerfall quantitativ erst in den Grundzustand übergeht, oder ob auch ein direkter $\beta$-Übergang in $\mathrm{Pd}$ stattfindet.

\footnotetext{
2 B. Pontecorvo, Physic. Rev. 54, 542 [1938].

3 E. C. Crittenden jr., Physic. Rev. 56, 709 [1939].
} 
Zur Untersuchung einer sehr weichen Komponente ist es nötig, die Präparate in sehr dünner Schicht zu verwenden, weil sonst die weiche Komponente gegenüber der noch vorhandenen harten $\beta$ Strahlung so stark benachteiligt wird, daß sie unmeßbar ist. Daher wurden Rhodiumpräparate einer Dicke von rund $1 \mathrm{mg} / \mathrm{cm}^{2}$ benutzt, die durch Kathodenzerstäubung auf einer $2 \mathrm{~mm}$ dicken Graphitunterlage niedergeschlagen waren ${ }^{4}$. Besondere Versuche hatten ergeben, daß bei unseren Bestrahlungsbedingungen diese Unterlage keine Aktivität zeigte. Zum Nachweis der Strahlung wurde ein Fensterzählrohr mit einer $20 \times 40 \mathrm{~mm}$ großen Zaponlackfolie von ${ }^{1 / 2} \mu$ Dicke benutzt. Die Anord-

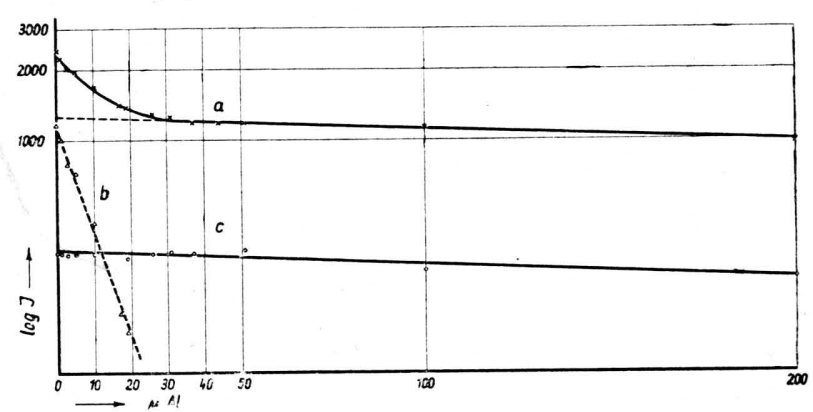

Abb. 1. Absorptionskurve der Strahlung der RhodiumAktivitäten in Aluminium:

a) ${ }^{104} \mathrm{Rh} *(4,3 \mathrm{~min})+{ }^{104} \mathrm{Rh}$ (42 se 2 Folgeprodukt).

b) Weiche Komponente allein. c) ${ }^{104} \mathrm{Rh}$ (42 sec).

nung war so getroffen, daß die aktivierten Präparate mitsamt der Absorptionsfolie mit Hilfe einer Vakuumschleuse vor das Zählerfenster gebracht werden konnten. Die Präparate wurden je 5 min mit verlangsamten $(\mathrm{Ra}+\mathrm{Be})$-Neutronen bestrahlt und nach Abklingen der 42-sec-Komponente hinter variablen Absorbern insgesamt 20 min gemessen. Zur Vergrößerung der Genauigkeit wurden viele Versuche identisch ausgeführt und addiert ${ }^{5}$.

Ergebnisse: Die erhaltene Absorptionskurve zeigt Kurve a der Abb.1, die deutlich zwei Komponenten erkennen läßt. Die härtere Komponente entspricht den $\beta$-Strahlen des Übergangs ${ }^{104} \mathrm{Rh} \rightarrow{ }^{104} \mathrm{Pd}$. Darüber gelagert ist die bereits von Pontecorvo gefundene weiche Komponente, die nach unseren Versuchen eine maximale Reichweite

4 Für die Herstellung der Rhodium-Niederschläge auf Graphit sind wir Hrn. Dr. Hiesinger (Physik. Untersuchungslabor, Onstmettingen) sehr $\mathrm{zu}$ Dank verpflichtet.

5 A. Flam mersfeld, Z. Naturforschg. 1, 3 [1946]; Naturwiss. 32, 36*[1944]. von $33 \mu \mathrm{Al}$ hat, entsprechend einer Energie der Elektronen von $87 \mathrm{KeV}^{\text {5a }}$; Kurve b zeigt noch die weiche Komponente nach Abzug der harten Komponente. Zur eindeutigen Zuordnung der weichen Komponente wurden noch ähnliche Versuche mit der kurzen 42-sec-Periode angestellt; die erhaltene Absorptionskurve zeigt Kurve c von Abb.1, in der keinerlei weiche Komponente erkennbar ist, so daß diese eindeutig zur langen Halbwertszeit gehört.

Der hier gefundene Wert der Energie der Elektronen ist nicht unwesentlich höher als der von Pontecorvo angegebene ( 35 bis $60 \mathrm{KeV}$ ), was wir auf die hier erzielte größere Genauigkeit zurückführen. Eine weitere Messung liegt vor von Ollano ${ }^{6}$, welche $69 \mathrm{KeV}$ angibt; doch war uns letztere Arbeit leider nicht zugänglich.

Von besonderem Interesse hinsichtlich der Frage des direkten $\beta$-Übergangs ist die Intensität der weichen Komponente. Denn da die harte Komponente die nach dem isomeren Übergang ausgesandten $\beta$-Strahlen darstellt, kann man aus dem Intensitätsvergleich beider Komponenten bei der Absorberdicke Null direkt die Zahl der Sekundärelektronen des isomeren Übergangs pro $\beta$-Zerfall ablesen. Doch sind hierbei noch folgende Fehler zu beachten bzw. folgende Korrektionen anzubringen:

1. Trotz der sehr geringen Dicke der Rhodiumschicht $\left(1 \mathrm{mg} / \mathrm{cm}^{2}\right)$ findet natürlich eine merkbare Selbstabsorption in der Präparatschicht statt. Aus der gemessenen Absorbierbarkeit der Elektronen in Aluminium und unter der Annahme der Massenproportionalität berechnet sich für diese Schicht eine Ausbeute von 85\%. Für die härtere Komponente ist die Präparatschicht natürlich so dünn, daß keine Selbstabsorption stattfindet.

2. Beide Komponenten könnten durch Rückstreuung verfälscht sein. Durch die verwendete leichtatomige Unterlage ist diese möglichst herabgesetzt; es lassen sich aber keine sicheren Angaben hierüber machen. Aus der Kurve der reinen 42-sec-Aktivität und aus zahlreichen weiteren Absorptionsversuchen mit anderen Substanzen läßt sich jedenfalls entnehmen, daß die Rückstreuung bei energiereichen $\beta$-Strahlen keine weichen Komponenten vortäuscht. Trotzdem wird ein gewisser Betrag an Rückstreuung in beiden Komponenten

5a A. Flammersfeld, Naturwiss. 33, 280 [1946].

6 Ollano, Ric. sci. Progr. tecn. Econ. naz. Scient. 11, 568 [1940], zitiert nach G. T. Seaborg, Rev. Mod. Physics 16, 1 [1944]. 
mitgemessen werden; wie groß ein etwaiger Unterschied bei beiden ist, ließ sich nicht ermitteln.

3. An der weichen Komponente sind wahrscheinlich einige Auger-Elektronen beteiligt; da die Fluoreszenzausbeute bei Rhodium etwa 75 bis $78 \%$ beträgt $^{7}$, können etwa $25 \%$ A uger-Elektronen anwesend sein. Aus der geschätzten Absorbierbarkeit dieser Elektronen, die eine Energie von rund $16 \mathrm{KeV}$ haben dürften, ergibt sich, daß in der Kurve der weichen Komponente etwa 7\% A uger-Elektronen enthalten sein könnten. Diese brauchen in der Kurve nicht als besondere weiche Komponente aufzutreten, da die eigentliche Absorptionskurve der 87-KeV-Elektronèn wegen der Homogenität ja am Anfang horizontaler verlaufen kann.

4. Bei der Beurteilung der Intensität der harten $\beta$-Komponenten ist folgendes $\mathrm{zu}$ beachten: Nach dem vorausgesetzten und durch unsere Messungen bestätigten Termschema zerfällt die 4,3-min-Aktivität $\left(T_{1}\right)$ mit dieser Halbwertszeit erst in den Grundzustand des ${ }^{104} \mathrm{Rh}$, welcher dann seinerseits mit der Halbwertszeit $42 \sec \left(T_{2}\right)$ unter Aussendung der harten $\beta$-Strahlen in ${ }^{104} \mathrm{Pd}$ übergeht. Die harte Komponente befindet sich also im laufenden Gleichgewicht mit der 4,3-min-Aktivität, und ihre Intensität erscheint in der Kurve um den Faktor $\frac{T_{1}}{T_{1}-T_{2}}$ zu hoch.

5. Andererseits ist $\mathrm{zu}$ berücksichtigen, daß das verwendete Zählrohr (18 mm $\varnothing, 5 \mathrm{~cm}$ Argon + Alkohol) wohl für die weiche Komponente $100 \%$ Ansprechwahrscheinlichkeit zeigen dürfte, nicht aber für die härtere Komponente. Nach Messungen von T. Graf ${ }^{8}$, J. De Vries und G. J. Sizoo ${ }^{9}$, J. W. Milatz u. H. Ten Kate ${ }^{10}$ liegen für harte $\beta$-Strahlen bei den üblichen Zählrohrbedingungen die Ansprechwahrscheinlichkeiten zwischen 70 und $100 \%$. Zur Prüfung unșerer Apparatur wurde der Gasdruck im Zählrohr variiert und die von einem Uran-Standard hinter $100 \mu \mathrm{Al}$ ausgehenden $\beta$-Strahlen gezählt. Diese $\beta$-Strahlen dürften etwa dasselbe Spektrum besitzen wie die 43-sec-Aktivität des Rhodiums. In Übereinstimmung mit den genannten Autoren ergab sich, daß bei unseren Versuchen, wo der Druck aus technischen Gründen nicht höher gewählt werden konnte, die Ansprech-

\footnotetext{
' Siehe W. Bothe, Handbuch der Physik 23, 2 [1933].

s T. Graf, Journ. Physique Radium 10, 513 [1939].

9 J. de Vries u. G. J. Sizoo, Physica 6, 593 [1939].
}

wahrscheinlichkeit etwa $90 \%$ betrug. Demgemäß ist also die harte Komponente zu erhöhen, um die wahre Intensität zu ergeben.

Unter Berücksichtigung aller dieser Korrektionen ergibt sich folgender Intensitätsvergleich beider Komponenten: Die Intensität der harten Komponente beträgt 1170 , die der weichen 1210 , was also sehr gute Übereinstimmung bedeutet, so daß auf jedes $\beta$-Teilchen des $\beta$-Zerfalls ein Elektron des Isomerenübergangs ausgesandt wird. Daraus ist erstens zu folgern, daß beim. ${ }^{104} \mathrm{Rh}^{*}$ der Übergang in den Grundzustand praktisch nur durch Aussendung eines Umwandlungselektrons erfolgt, daß also 100-proz. Umwandlung des betreffenden $\gamma$-Strahls stattfindet. Zweitens folgt, daß kein direk-

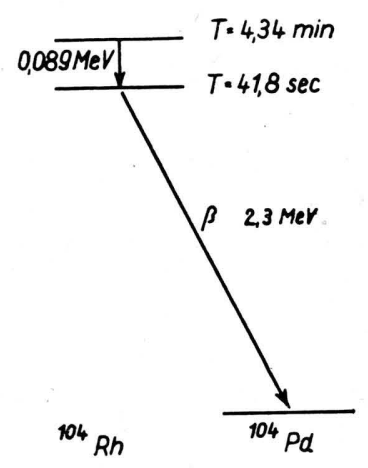

Abb. 2. Niveauschema des ${ }^{104} \mathrm{Rh}$. Statt $0.089 \mathrm{MeV}$ muß es heißen $0.087 \mathrm{MeV}$.

ter $\beta$-Übergang vom angeregten ${ }^{104} \mathrm{Rh}^{*}$ zum ${ }^{104} \mathrm{Pd}$ stattfindet, denn sonst müßten mehr $\beta$-Teilchen als Umwandlungselektronen auftreten.

Die Energie der Elektronen des Isomeren-Übergangs beträgt $87 \cdot \mathrm{KeV}$. Aus dieser Energie und der Halbwertszeit läßt sich auf einen Drehimpulsunterschied zwischen angeregtem Zustand und Grundzustand von etwa $\Delta i=4$ schließen. Bei derartig hohen Spindifferenzen findet aber nach den Rechnungen von $\mathrm{Hebb}$ und Nelson ${ }^{11}$ und Flügge ${ }^{12}$ die Auslösung der Elektronen nicht mehr bevorzugt in der $K$-Schale statt, sondern u. U. ist die Auslösung in der $L$-Schale stärker, und auch die $M$ - und $N$-Schalen können noch nennenswerte Beiträge liefern. Nach den genannten Auto-

\footnotetext{
10 J. W. Milatz u. H. Ten Kate, Physica 7, 779 [1940].

11 M. H. Hebbu. E. Nels on, Physic. Rev. 58, 486 [1940].

12 S. Flüg g e, Physik. Z. 42, 221 [1941].
} 
ren ist das Verhältnis $\frac{N_{K}}{N_{L}}$ der in der $K$-Schale zu den in der $L$-Schale ausgelösten Elektronen für den vorliegenden Fall etwa zu 0,5 anzunehmen. Der größte Teil der Umwandlungselektronen stammt also aus der $L$-Schale, und da auch die $(M+N)$ Schalen beitragen, ist zur gemessenen Elektronenenergie nur die Ablösearbeit der letzten hinzuzuzählen, um die gesuchte Anregungsenergie zu ergeben. Da die Ablösearbeit zu vernachlässigen ist, beträgt also die Anregungsenergie des isomeren Zustandes beim ${ }^{104} \mathrm{Rh}^{*} E=87 \mathrm{KeV}$. Das mit den erhaltenen Resultaten sich ergebende Niveauschema zeigt Abb. 2.

\section{2. $103 \mathrm{Rh} *$}

${ }^{103} \mathrm{Rh}^{*}$, ein Isomer zum stabilen ${ }^{103} \mathrm{Rh}$, wurde zuerst von einem von uns durch Anregung mit schnellen $(D+D)$-Neutronen dargestellt ${ }^{5}$. Bei den früheren Versuchen wurden Rhodium-Bleche von 30 und $100 \mu$ Dicke bestrahlt, wobei im $10-\mu$-Zähler eine Aktivität von $T=48 \pm 5$ min Halbwertszeit festgestellt werden konnte, die eine reine Quantenstrahlung aussandte. Deren Intensität schien verhältnismäßig schwach zu sein, so daß keine näheren Angaben gemacht wurden.

Bei der Durchführung der beim ${ }^{104} \mathrm{Rh}^{*}$ beschriebenen Versuche zeigte sich zuerst eine weitere, sehr weiche Komponente von Elektronen, die nicht erklärt werden konnte. Bei der Suche nach ihrem Ursprung konnte festgestellt werden, daß sie weder zur 42-sec- noch zur 4,3-min-Aktivität gehörte, sondern zu einer längerlebigen Substanz, die bei dem verwendeten, sehr dünnen Zählerfenster ohne Absorber mit unvermutet großer Intensität auftrat. Bestrahlungen von mehreren Stunden mit schnellen Neutronen ergaben eine Anfangsaktivität von rund $300 / \mathrm{min}$, die mit einer Halbwertszeit von $52 \pm 2$ min abfielen. Die Aktivität war nicht verstärkbar, denn Umgeben der Quelle mit Paraffin war ohne Einfluß auf die Intensität. Die ausgesandte Strahlung ist extrem weich, die Halbwertsdicke beträgt $1,4 \mu$, und die aus Intensitätsgründen nur mäßig genau bestimmbare Reichweite beträgt $8 \pm 1 \mu \mathrm{Al}$, was einer Energie von $42 \mathrm{KeV}$ entspricht. Zur besseren Festlegung dieser Energie wurde noch eine Absorptionskurve der vom 4,4-Std.-Brom ausgesandten 47-KeV-Elektro- nen aufgenommen, die eine Reichweite von $10 \mu \mathrm{Al}$ besitzen.

Die gefundene Aktivität ist zweifellos mit der früher angegebenen $T=48$-min-Aktivität identisch, da sie wie diese mit schnellen Neutronen durch (n-n)-Anregung entsteht und aus Betrachtungen im Sargent-Diagramm, ähnlich wie beim 1,25min-Dysprosium und beim 19 -sec-Hafnium ${ }^{13}$, zweifellos hervorgeht, daß sie keine $\beta$-Aktivität sein kann. Ein $\beta$-Zerfall von 52 min Halbwertszeit sollte eine Energie von mindestens $500 \mathrm{KeV}$ oder ein $\beta$ Zerfall der Energie $43 \mathrm{KeV}$ mindestens eine Halbwertszeit von 2 Jahren aufweisen. Also ist auch hier wieder zu schließen, daß es sich bei der Aktivität um einen Isomeren-Übergang handelt; der Übergang in den Grundzustand findet durch Aussendung von Sekundärelektronen an Stelle von $\gamma$ Strahlung statt.

Wie im vorhergehenden Fall muß aus der Energie und der Halbwertszeit auf einen Drehimpulsunterschied von $\Delta i=4$ geschlossen werden, woraus sich nach Hebb u. Nelson ${ }^{11}$ und Flügge ${ }^{12}$ $N_{K} / N_{L} \approx 0,2$ ergibt. Daher ist wieder zur Energie der Elektronen nur die Ablösearbeit der $(M+N)$ Schale hinzuzuzählen, die zu vernachlässigen ist, und es ergibt sich für die Anregungsenergie des metastabilen Zustandes ${ }^{103} \mathrm{Rh}^{*} E=42 \mathrm{KeV}$. Daß bei den früheren Versuchen die weichen Elektronen nicht gefunden wurden, liegt daran, daß damals nur ein Zählrohr mit einem Glimmerfenster von $10 \mu$ Dicke zur Verfügung stand, welches die Elektronen nicht durchdringen konnten. Die damals gefundene Quantenstrahlung stellt keine $\gamma$-Strahlung dar, sondern die $K$-Strahlung aus der nach der Umwandlung zurückbleibenden $K$-Schale. Die Absorptionsversuche der Quantenstrahlung ergaben in $\mathrm{Al}$ ein $\mu / \varrho \approx 8$, was gut zu der zu erwartenden $K$-Strahlung $(20 \mathrm{KeV})$ paßt. Bemerkenswert ist noch der große Wirkungsquerschnitt für den $(n, n)$-Prozeß beim Rhodium; er beträgt für unverlangsamte $(\mathrm{Ra}+\mathrm{Be})$-Neutronen mindestens das 20-fache des Wirkungsquerschnittes für Neutroneneinfang (Entstehung der 4,3-min-Aktivität) und ist also in derselben Größenordnung wie der für den $(n, n)$-Prozeß beim Silber, nämlich einige $10^{-24} \mathrm{~cm}^{2}$.

13 A. Flammersfeld, Naturwiss. 32, 68 [1944]; Z. Naturforschg. 1, 190 [1946]. 